\title{
В.Е. Плюгин
}

\section{КЛАССОВАЯ СТРУКТУРА МОДЕЛЕЙ ЭЛЕКТРОМЕХАНИЧЕСКОГО ПРЕОБРАЗОВАТЕЛЯ ЭНЕРГИИ С ИСПОЛЬЗОВАНИЕМ UМL-ДИАГРАММ}

\author{
Приведені результати теоретичних досліджень, показано формування математичної моделі довільного електроме- \\ ханічного перетворювача енергії шляхом наслідування від узагальненого класового шаблону.
}

Приведены результаты теоретических исследований, показано формирование математической модели произвольного электромеханического преобразователя энергии путем наследования от обобщенного классового шаблона.

Строгая постановка такой сложной проблемы, как пояснение принципов формообразования и структурообразования объектов электромеханики невозможна без наличия научно обоснованной классификации их порождающих структур. Определение признаков и принципов построения такой классификации составляет отдельную проблему системного характера [1].

Фактическое отсутствие научно-обоснованных подходов, которые можно было бы положить в основу структурной классификации электромеханических преобразователей энергии (ЭМПЭ), привела к тому, что в электромеханике существует путаница в вопросах классификации большого количества структурных и функциональных разновидностей электрических машин (ЭМ).

Структурно-системные исследования, которые в последнее время проводятся в направлении создания обобщенной теории физических структур, определения закономерностей общего развития технических систем в целом, не могут быть использованы для решения фундаментальных и прикладных задач электромеханики, так как они имеют общий характер и не учитывают особенностей структурной организации и специфики электромагнитных процессов, определяющих функционирование и поведение электромеханических систем (ЭС).

Так, электрические машины разделяют на синхронные и асинхронные, беря за основу классификации соотношение между угловыми скоростями ротора $\omega_{\text {r }}$ и поля статора $\omega_{c}$. Электрические машины делят по роду питания [2]. Надо ли говорить, что такая классификация является условной? Ведь одна и та же машина может работать и как синхронная, и как асинхронная, а при наличии выпрямителей машину постоянного тока можно использовать в сети переменного тока. Не более удачным является классификация ЭМ по их математическим моделям, а именно по числу уравнений, отражающих процессы электромеханического преобразования энергии [2]. Так, для определенного числа полей и числа обмоток на статоре и роторе приводятся уравнения напряжений и пары произведений токов в статоре и роторе в уравнении электромагнитного момента. По сформированной таблице "можно для машин с определенным числом обмоток и гармоник в воздушном зазоре представить сложность уравнений, оценить необходимое время для программирования". Также утверждается, что классификация ЭМПЭ по виду их математических моделей "позволяет накопить стандартные программы для ЭВМ и сравнительно быстро определить динамические и статические характеристики для задач электромеханики, не прибегая всякий раз к составлению уравнений и программ".

Однако, приведенные выше выдержки из работы [2] можно считать скорее внутренним содержанием объ- ектов электромеханики (и в этом плане к ним замечаний нет), способом их математического описания, но никак не средством классификации. Математическая модель это лишь абстрактное представление реальных объектов, способная замещать его в определенных отношениях, дающая при ее исследовании, в конечном счете, информацию о самом моделируемом объекте.

В последние годы интенсивно развивается информационная технология, особенно в направлении исследований, называемых искусственным интеллектом.

Общие недостатки, как традиционных информационных систем, так и методов программного представления расчета, моделирования и проектирования ЭМПЭ, заключаются в слабой адаптивности к изменениям в предметной области и информационным потребностям пользователей, в невозможности решать плохо формализуемые задачи, с которыми проектировщики постоянно имеют дело: анализ проблемных ситуаций, возникающих в расчетных процессах, прогноз дальнейшего использования и надежности, принятие решений и выбор оптимального варианта в задачах оптимизации. Для решения подобных задач разрабатываются и используются системы искусственного интеллекта.

Более того, существующая классификация и алгоритмы расчета ЭМПЭ базируются на устаревших принципах, так называемых "процедурных" языках программирования. Современные объектно-ориентированные языки программирования и программные платформы разработки программного обеспечения нуждаются в изменении принципов классификации и структурообразования ЭМПЭ.

В данной работе рассматриваются модели, методы и средства разработки программных систем ЭМПЭ на основе методологии объектно-ориентированного анализа и проектирования [3-5].

Основная идея объектного подхода состоит в том, чтобы заключить данные и связанные с ними процедуры в некие структуры (объекты), объединенные механизмом наследования. Объектно-ориентированные подход к моделированию и проектированию программных систем наилучшим образом подходит для решения проблем, требующих детального представления объектов реального мира и динамических отношений между ними. В таких программах компоненты сложной системы представляются структурами, инкапсулирующими и данные, и функции, моделирующие поведение соответствующих компонентов.

Основные идеи объектно-ориентированного подхода опираются на следующие положения:

- программа представляет собой модель некоторого реального процесса, части реального мира; 
- модель реального мира или его части может быть описана как совокупность взаимодействующих между собой объектов;

- объект описывается набором параметров, значения которых определяют состояние объекта, и набором операций (действий), которые может выполнять объект;

- взаимодействие между объектами осуществляется посылкой специальных сообщений от одного объекта к другому. Сообщение, полученное объектом, может потребовать выполнения определенных действий, например, изменения состояния объекта;

- объекты, описанные одним и тем же набором параметров и способные выполнять один и тот же набор действий, представляют собой класс однотипных объектов.

Философия представления знаний о реальном мире в терминах взаимодействия объектов и субъектов предоставляет достаточно удобную среду для решения большого класса задач моделирования и проектирования сложных систем, в том числе систем искусственного интеллекта. Методика представления абстрактных данных и процедур позволяет разработчикам на ранних этапах сосредоточиться на выборе подходящих видов объектов и их поведении, не вдаваясь в подробности реализации функций и структур данных.

Методология объектно-ориентированного анализа и проектирования получила широкое распространение с появлением языка объектного моделирования нового поколения - унифицированного языка моделирования Unified Modeling Language (UML), предназначенного для визуального моделирования и проектирования информационных систем [6]. Применение современных средств моделирования позволяет реализовать такие методы системного анализа, как создание иерархии понятий, обобщение понятий, наследование свойств, многообразие моделей описания предметной области, визуализацию представлений о процессах, протекающих в рассматриваемой предметной области.

В связи с этим значение языка UML существенно возрастает, поскольку он все более приобретает черты языка представления знаний. При этом, наличие в языке UML изобразительных средств для представления структуры и поведения модели, позволяет достичь адекватного представления декларативных и процедурных знаний и, что не менее важно, установить между этими формами знаний семантическое соответствие. Все эти особенности языка UML позволяют сделать вывод о том, что он имеет самые серьезные перспективы в качестве средства разработки моделей представления знаний.

Классовая структура математической модели обобщенного ЭМПЭ представлена в виде UMLдиаграммы на рис. 1.

Особенностью диаграммы, показанной на рис. 1 является то, что она выступает в роли некоего шаблона, а говоря в терминах программирования, класса обобщенной электрической машины. Другими словами, изначально отсутствует деление ЭМПЭ по какимлибо признакам. Кроме того, ключевыми словами являются "класс" и "обобщенный". Диаграмма не является сущностью, замещающей реальную машину это инструмент для генерации множества объектовмашин посредством предоставляемых инструментов наследования и выбора.

На диаграмме (рис. 1) блок атрибутов является матрицей параметров обобщенной модели ЭМПЭ, записанной в представленном случае, для двухфазной системы координат. Обобщенная модель, характерная для любых типов машин, уточняется посредством блоков, расположенных ниже. Блок дополнительных атрибутов представляет собой набор модификаторов параметров цепей статора и ротора в системе дифференциальных уравнений [2]. Блок напряжений отвечает за источники питания первичной и вторичной цепей машины. Связанные блоки функции вращения и событий являются модификаторами вращения вторичного элемента. Наконец, набор атрибутов с подмножеством ассоциаций формирует требования ко вторичному элементу машины.

Пользуясь классовым шаблоном обобщенного ЭМПЭ (рис. 1) и выбирая определенные признаки, можно перейти к объекту конкретного ЭМПЭ. Так, на рис. 2 показана уже не классовая, а объектная диаграмма асинхронного двигателя с короткозамкнутым ротором, порожденного из классовой диаграммы рис. 1.

На рис. 3 показана диаграмма, аналогичная приведенной на рис. 2, но с обозначениями, присущими для математических моделей, записанных в дифференциальной форме. Так, показанная на диаграмме таблица с занесенными в нее модификаторами, является ничем иным, как матрицей параметров в системе дифференциальных уравнений обобщенного ЭМПЭ.

Алгоритм формирования объектной модели показан на рис. 4. Эта диаграмма носит названия диаграммы состояний. Модель состояний описывает последовательности операций, происходящих в системе в ответ на внешние воздействие (в противоположность содержанию, предмету и реализации операций, описываемых моделью классов) [6].

Диаграммы на рис. 2, 3 являются непосредственным воплощением асинхронного двигателя с короткозамкнутым ротором в виде абстрактной модели, по которой можно выполнять расчеты. Аналогичным образом, можно порождать объектные модели как известных видов ЭМПЭ, так и еще не созданных, наделяя их новыми признаками.

В настоящее время программные продукты и средства моделирования и проектирования электрических машин представлены преимущественно объектноориентированными языками программирования, такими как Visual Basic, Delphi, C++, C\#, Eiffel, Oberon, Java. B то же время, описанные в многочисленных учебниках и справочной литературе алгоритмы, последовательности расчета электрических машин идеологически соответствуют устаревшему и изжившему себя процедурному подходу в программировании. Разработчик программного продукта выполняет лишний шаг, сначала переделывая процедурную методику в объектную, а затем объектную в программный код.

Целью последующих исследований автора является приведение в соответствие расчетных методик электромеханических преобразователей энергии их программному отображению согласно объектноориентированной концепции. Ведь, несмотря ни на что, машины рассчитываются, и будут рассчитываться не вручную, по формулам, а в объектноориентированных программах. Поэтому, представляется актуальной проблема именно объектноориентированного расчета и моделирования электрических машин. 


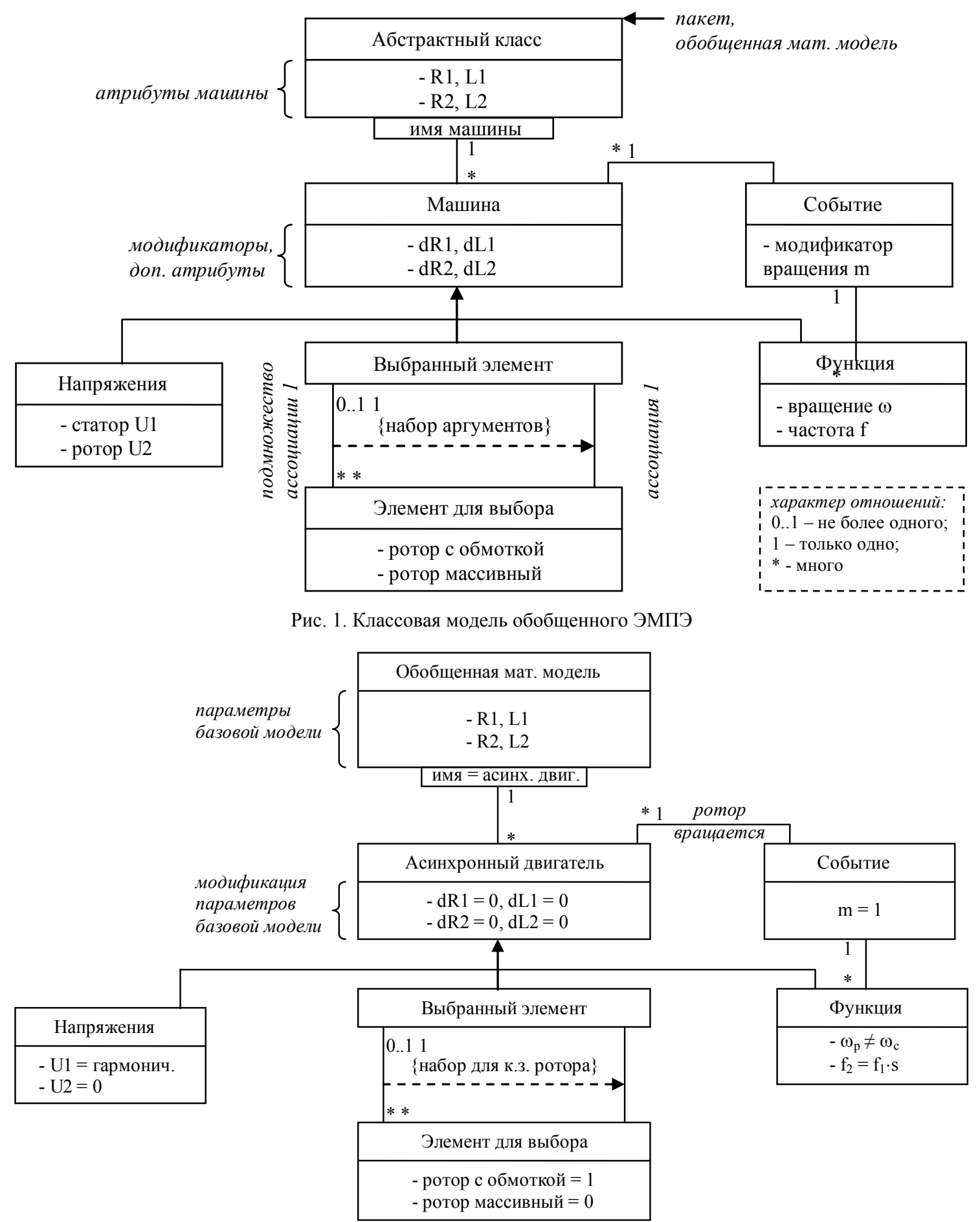

Рис. 2. Диаграмма объекта: асинхронный двигатель с к.з. ротором

ВЫВОДЫ

1. Проанализированы существующие подходы к классификации разновидностей ЭМ и доказана их несостоятельность в отношении адекватного отражения картины реального мира. В качестве инновационного метода классификации видов ЭМ предложен подход, основанный синтезе моделей с использованием диаграмм UML.

2. Все многообразие ЭМПЭ представлено в виде диаграммы классов, содержащей атрибуты наследования, ограничения, событий выбора, элементов агрегации и композиции. Классовая диаграмма ЭМПЭ является абстрактным шаблоном для порождения объектных моделей конкретных видов ЭМПЭ.

3. Особенностью классового представления ЭМПЭ в виде универсальной диаграммы является отсутствие деления машин по каким-либо признакам. Наоборот, любую машину можно получить из универсальной диаграммы, задаваясь определенными предпосылками. Это касается как уже известных на сегодняшний день видов машин, так и еще не открытых.

4. Объектные модели, порожденные от класса обобщенного ЭМПЭ, обладают признаками, присущими конкретному виду ЭМПЭ. Объектная модель приводит к формированию математической модели ЭМПЭ, готовой к расчетам. 
Обобщенная мат. модель

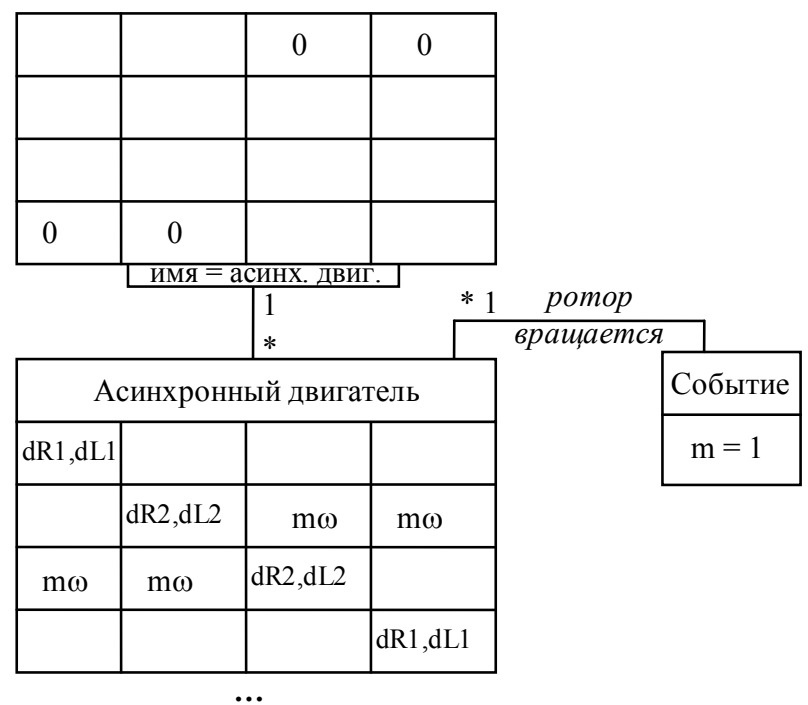

Рис. 3. Фрагмент объектной модели асинхронного двигателя
СПИСОК ЛИТЕРАТУРЫ

1. Шинкаренко В.Ф. Основи теорії еволюції електромеханічних систем. - К.: Наукова думка, 2002. -288 с.

2. Копылов И.П. Математическое моделирование электрических машин: учеб. для студ. ВУЗов. - М.: Высшая школа, 2001. $-327 \mathrm{c}$.

3. Шинкаренко В.Ф., Заблодский Н.Н., Плюгин В.Е. Обоснование принципов объектно-ориентированного проектирования электромеханических преобразователей энергии // Вісник НТУ "ХПІ". - 2011. - №48 - С. 76-83.

4. Заблодский Н.Н., Плюгин В.Е. Объектноориентированное проектирование электромеханических преобразователей энергии с совмещенными функциями // Сб. наук. праць ДонДТУ. Алчевськ: ДонДТУ, ВПЦ "Ладо". - 2011. - №34. - С. 321-327.

5. Плюгин В.Е. Математическая модель электромеханического преобразователя энергии с массивным ротором // Електротехніка і електромеханіка. - 2012. - №1. - С. 42-44.

6. Рамбо Дж., Блаха M.. UML 2.0. Объектноориентированное моделирование и разработка. - СПб.: Питер, 2007. - $544 \mathrm{c}$.

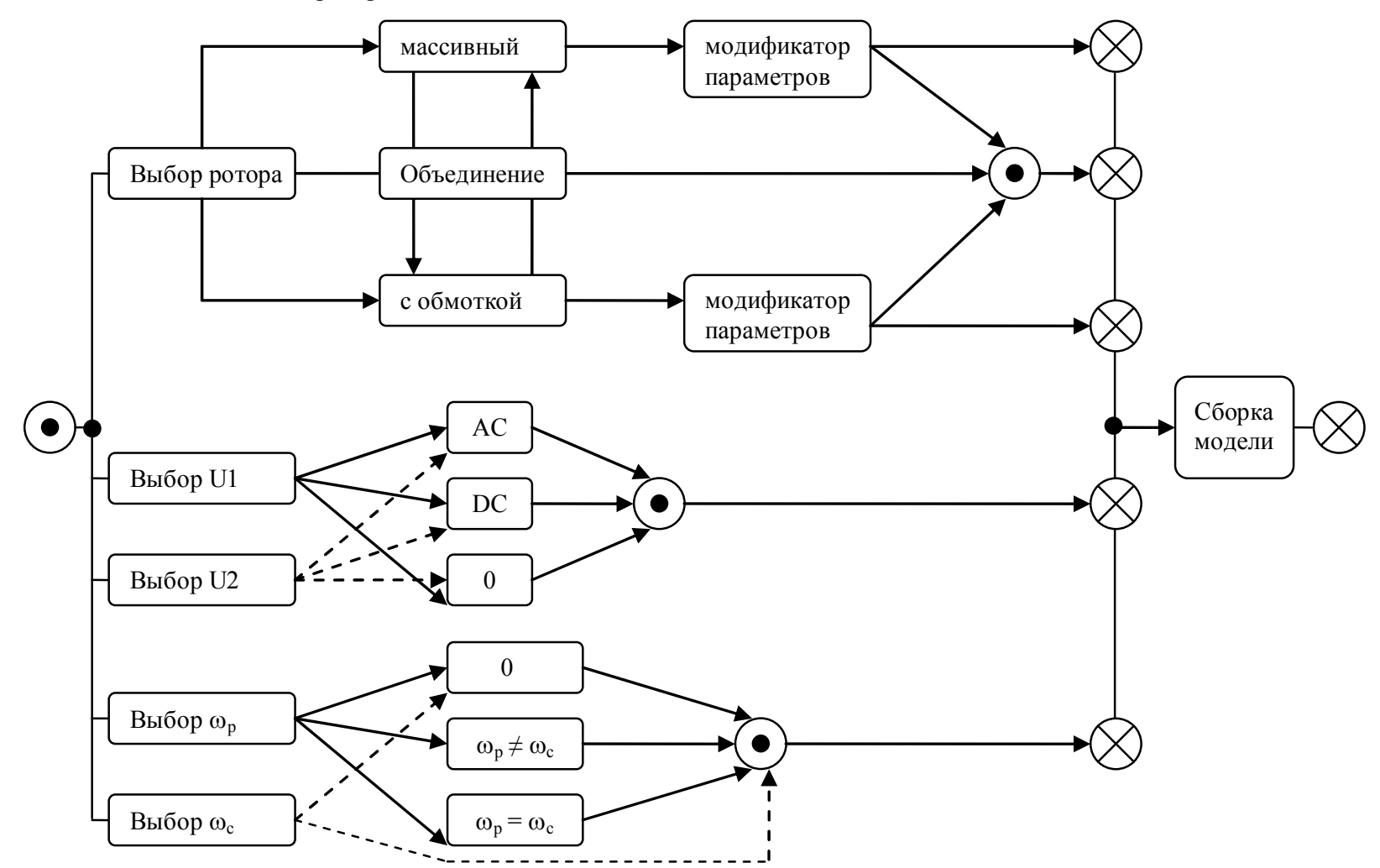

Рис. 4. Диаграмма состояний формирования модели асинхронного двигателя с к.з. ротором

Bibliography (transliterated): 1. Shynkarenko V.F. Osnovy teorii evoliutsii elektromekhanichnykh system. Kyiv, Naukova dumka Publ., 2002. 288 p. 2. Kopylov I.P. Matematicheskoe modelirovanie jelektricheskih mashin: High school book. Moscow, Vysshaja shkola Publ., 2001. 327 p 3. Shynkarenko V.F., Zablodskiy N.N., Plyugin V.Ye. Substantiation of principles of object-oriented designing in electromechanical energy converters. Bulletin of NTU "KhPI", 2011, no.48, pp. 76-83. 4. Zablodskiy N.N., Plyugin V.Ye. Ob'ektno-orientirovannoe proektirovanie jelektromehanicheskih preobrazovatelej jenergii s sovmeshhennymi funkcijami. $S b$. nauk. prats DonDTU. Alchevsk: DonDTU, Lado Publ., 2011, no.34, pp 321-327. 5. Plyugin V.Ye. A mathematical model of an electromechanical energy converter with a massive rotor. Electrical engineering \& electromechanics, 2012, no.1, pp. 42-44. 6. Rambo Dzh., Blaha M.. UML 2.0. Ob'ektno-orientirovannoe modelirovanie i razrabotka. St.Petersburg, Piter Publ., 2007. 544 p.

Поступила (received) 18.10.2013
Плюгин Владислав Евгеньевич, к.т.н., дои.,

Донбасский государственный технический университет, 94204, Алчевск, пр. Ленина, 16,

тел/phone +38050 2021045, e-mail: vlad.plyugin@gmail.com

V.Ye. Plyugin

Donbass State Technical University

16, Lenin Avenue, Alchevsk, Lugansk region, 94204, Ukraine

Class structure of electromechanical energy converter models with UML-diagrams application.

Theoretical results are presented, mathematical model formation by means of inheritance from a generalized class template is shown for a general electromechanical energy converter.

Key words - object-oriented, design, electrical machine, class, object, mathematical model, inheritance, template. 